\title{
Chemical Reaction Effects On Mhd Mixed Convection Flow Of Water At Maximum Density Past A Vertical Plate Under Variable Temperature
}

\author{
Sarat Sharma ${ }^{1}$ and Rudra Kanta Deka ${ }^{2}$ \\ 1 Department of Mathematical Sciences, Chaiduar College, Gohpur (Assam), India-784168 \\ 2 Mathematics Department, Gauhati University, Guwahati (Assam),India-7840014
}

\begin{abstract}
Flow of water at its maximum density past a vertical plate is considered. Effects of a transversely applied magnetic field, variation of the plate temperature and a first order chemical reaction on the flow field are studied by a similarity transformation of the governing equations. For various values of the magnetic, plate temperature exponent and chemical reaction parameter, numerical values proportionate to Skin friction, Nusselt number and Sherwood number are tabulated and graphical results for the velocity, temperature and concentration profiles are presented. Computed values and graphical results for flow of water at a normal temperature are compared with that of flow at $\mathbf{4}^{0} \mathrm{C}$.
\end{abstract}

Key words: Chemical reaction, density of water, plate temperature, Prandtl number, Schmidt number.

\section{Introduction:}

Flow of electrically conducting fluid past a vertical plate is a widely studied problem in fluid dynamics. The fact that magnetic field has profound influence on boundary layer flow of an electrically conducting fluid, has attracted the attention of researchers due to its various applications in plasma physics, nuclear science, engineering design and space dynamics. Such flows are termed as MHD flows in the broader sense and abundant literature reviews are available concerning MHD convective flow of fluid past a vertical plate.

Convection heat transfer coefficients, natural or forced, are a strong function of the fluid velocity. In dealing with forced convection flow we generally ignore the effects of free convection. Similarly we assume the forced convection as negligible while dealing with free convection flow. The error involved in ignoring natural convection while studying forced convection is negligible at high velocities but may be considerable at low velocities. Situations may arise for which free and forced convection effects are comparable, in which case it is inappropriate to neglect either process. Therefore it is desirable to have a criterion to assess the relative magnitude of natural convection in the presence of forced convection flows. Such flows situations where both free and forced convection effects are of comparable order belong to the mixed convection regime. In several practical applications of heat transfer theory to the vertical plate problems there exists significant temperature difference between the surface of the hot plate and the free stream. This temperature difference cause density gradients in the fluid medium and in the presence of a gravitational body force, free convection effects become important. It has generally been recognized that $\lambda=\frac{G r}{\mathrm{Re}^{2}}$, where $G r$ is the Grashof number and $R e$ the Reynolds number is the governing parameter for the laminar boundary layer forced-free mixed convection flow which represents the ratio of the buoyancy forces to the inertial forces inside the boundary layer. Forced convection exists when $\lambda \rightarrow 0$ which occurs at the leading edge and the free convection limit can be reached if $\lambda$ becomes large. We know from authoritative work in heat transfer [1] that free convection is negligible if $\lambda \leq 1$ and forced convection is negligible if $\lambda \geq 1$. Hence combined free and forced (or mixed) convection regime is generally one for which $\lambda \approx 1$. Although the flows with only one of the two effects involved will have a self similar character, such situations will mathematically lead to ordinary differential equations and are easy to solve. But as soon as the two effects occur together, boundary value problem involving partial differential equations will arise and are not easy to solve by conventional procedures. The physical explanation of the complexities is that the two effects act differently with respect to the characteristic length $l$ and any combination means that the characteristic length is introduced into the problem (i.e., the length from the leading edge to the separation position).If both the effects are to be considered, pure forced convection always dominates for $l \rightarrow 0$ while direct natural convection dominates for $l \rightarrow \infty$. Most of the works concerning external flows past a vertical plate are either considered for free or forced convection regimes only. However mixed convection flows received considerable attention in the late 1970s to 1980s and numerous literature reviews are available [2-4]. In nature it is rather impossible to find pure fluid unless special efforts are made to obtain it. The most common fluids like water, air etc. are contaminated with impurities like $\mathrm{CO}_{2}, \mathrm{C}_{6} \mathrm{H}_{6}, \mathrm{H}_{2} \mathrm{SO}_{4}$ etc. and generally we have to 
consider presence of such foreign mass while studying flows past different bodies. In such a case the density difference in the fluid is caused by material constitution in addition to temperature differences. The common example of such a flow is the atmospheric flow which is driven appreciably by both temperature and $\mathrm{H}_{2} \mathrm{O}$ concentration differences. When such contaminant is present in the fluid under consideration there does occur some chemical reaction e.g. air and benzene react chemically, so also water and sulfuric acid. During such chemical reactions, there is always generation of heat. But when the foreign mass present in the fluid at very low level, we can assume a first order chemical reaction and the heat generated due to chemical reaction can be very negligible. Several authors have done significant works by taking into account a first order chemical reaction on flow past vertical surfaces [5-7].

Water is an intriguing substance that plays a vital role in sustaining life on earth. Despite being the most abundant liquid on earth, it is also the most anomalous. Some of these anomalous properties make water particularly well adapted for its various roles in sustaining life on the planet. When liquid water freezes, it becomes less dense. Contrary to the behavior of most other solids, water expands on freezing and the solid state is less dense than the liquid state. This behavior has a significant impact on aquatic life for keeping ice at the top of ponds and lakes during cold winters allowing aquatic life to survive at the bottom where warm water circulates. Another property of water that is exploited by aquatic life to survive under such cold conditions is the colligative nature of its freezing point. When a solute such as sodium chloride or sucrose is added to water the temperature at which it freezes is lowered. The physical properties of water such as the thermal conductivity, the specific heat capacity, the viscosity and the density are linear functions of temperature. A notable exception to this linear variation is liquid water which at a pressure of one atmosphere has a maximum density of 999.9720 $\mathrm{kg} / \mathrm{m}^{3}$ at $4^{0} \mathrm{C}$. Above this temperature, the density of water decreases as the temperature is increased in a manner similar to other fluids. For temperature below $4^{\circ} \mathrm{C}$ the trend is reversed, density increases with increased temperature giving rise to the maximum density at $4^{\circ} \mathrm{C}$. This type of parabolic variation of the density of water as a function of temperature is of fundamental importance to this study.

The driving mechanism of the convective flows is described by the buoyancy term in the momentum equation of the governing equations. As a consequence of this, the order of magnitude of all terms in this equation is dictated by the buoyancy term. However, this buoyancy term came up as a consequence of expanding the density in a Taylor series and taking only the linear term into account and ignoring all other terms as follows:

$$
\begin{aligned}
\rho(T)=\rho_{\infty}+\left(\frac{\partial \rho}{\partial T}\right)_{\infty} & \left(T-T_{\infty}\right)+\left(\frac{\partial^{2} \rho}{\partial T^{2}}\right)_{\infty} \frac{\left(T-T_{\infty}\right)^{2}}{2 !}+\left(\frac{\partial^{3} \rho}{\partial T^{3}}\right)_{\infty} \frac{\left(T-T_{\infty}\right)^{3}}{3 !}+\cdots \\
= & \rho_{\infty}\left[1-\left\{-\frac{1}{\rho_{\infty}}\left(\frac{\partial \rho}{\partial T}\right)_{\infty}\right\}\left(T-T_{\infty}\right)-\left\{-\frac{1}{\rho_{\infty}}\left(\frac{\partial^{2} \rho}{\partial T^{2}}\right)\right\}_{\infty} \frac{\left(T-T_{\infty}\right)^{2}}{2 !}-\cdots\right] \\
= & \rho_{\infty}\left[1-\beta_{\infty}\left(T-T_{\infty}\right)-\left\{-\frac{1}{\rho_{\infty}}\left(\frac{\partial^{2} \rho}{\partial T^{2}}\right)_{\infty}\right\} \frac{\left(T-T_{\infty}\right)^{2}}{2 !}-\cdots\right] \\
\frac{\rho(T)-\rho_{\infty}}{\rho_{\infty}} & =-\beta_{\infty}\left(T-T_{\infty}\right)-\beta_{\infty}^{*}\left(T-T_{\infty}\right)^{2}-\cdots
\end{aligned}
$$

Here $\beta_{\infty}=-\frac{1}{\rho_{\infty}}\left(\frac{\partial \rho}{\partial T}\right)_{\infty}$ is the coefficient of thermal expansion at temperature $T_{\infty}$. While invoking the Boussinesq approximation, it is generally assumed that $\beta_{\infty}$ does not vanish, but for water at $4^{0} \mathrm{C}$ the density is maximum and consequently $\beta_{\infty}$ becomes equal to zero. Considering this fact into account, Goren [8] has shown that for temperature sufficiently close to $T_{c}=3.98^{\circ} \mathrm{C}$, the relationship between fluid temperature $T$ and the density $\rho_{c}$ is given by the relation:

$$
\frac{\rho(T)-\rho_{c}}{\rho_{c}}=-\beta_{\infty}^{\star}\left(T-T_{c}\right)^{2}
$$

Where $\rho_{c}$ the maximum density of the liquid and $\beta_{\infty}^{*}=8.0 \times 10^{-6}(C)^{-2}$ is the thermal expansion coefficient of water at $4^{\circ} \mathrm{C}$. This type of parabolic variation of density has attracted the attention of many researchers and several authors have done significant theoretical and experimental work [9]-[18] taking up the flow of water at its maximum density. Presence of soluble species in water and chemical reaction effects on the flow of water at maximum density have not been undertaken in the literature so far, which motivated our study. We propose to study the effects of chemical reaction and a transversely applied magnetic field on the steady flow of water at $4^{\circ} \mathrm{C}$ past a vertical plate when the temperature of the plate has a power law variation. 


\section{Formulation Of The Problem}

A two-dimensional combined free-forced convective flow of water at its maximum density along a semiinfinite vertical isothermal flat plate in presence of a soluble species is considered. The flow is assumed to be in the direction of $x^{I}$-axis which is taken along the vertical plate in the upward direction and the $y^{I}$-axis is taken to be normal to the plate. Let $u^{I}$ and $v^{I}$ be the components of velocities of water along $x^{I}$ and $y^{I}$ axes respectively. Flow configuration is shown in Fig.1. A transverse magnetic field of uniform strength $B_{0}$ is applied normal to the flow and the induced magnetic field is neglected. The concentration of diffusing species at the plate is $C_{w}{ }^{\prime}$ and $C_{\infty}{ }^{\prime} \quad$ far from the plate. Initially the surface of the plate is at the same free stream temperature $T_{\infty}{ }^{\prime}$ and the uniform free stream velocity $u_{\infty}$ parallel to the vertical plate. The plate temperature and species concentration level near the plate are raised to $T^{\prime}{ }_{w}\left(T^{\prime}{ }_{w}>T^{\prime}>T_{\infty}^{\prime}\right)$ where we assume a power law variation of the plate temperature viz. $T^{\prime}{ }_{w}-T_{\infty}^{\prime}=A x^{n}$ and $C_{w}^{\prime}\left(C_{w}^{\prime}>C^{\prime}>C_{\infty}\right)$ respectively. We assume that there exists a homogeneous chemical reaction of first order with rate constant $k_{1}$ between the diffusing species and water. We neglect Dufour and Soret effects and viscous dissipation and assume all fluid properties as constant except variation of density and concentration in the body force term. Then making use of (1), under the usual Boussinesq approximation the boundary layer equations based on the conservation of mass, momentum and energy, governing the flow problem can be expressed as:

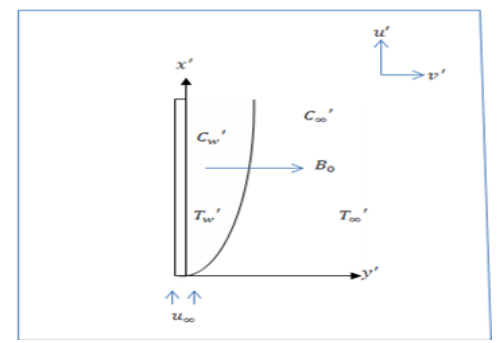

Figure 1

$$
\begin{aligned}
& \frac{\partial u^{\prime}}{\partial x^{\prime}}+\frac{\partial v^{\prime}}{\partial y^{\prime}}=0 \\
& u^{\prime} \frac{\partial u^{\prime}}{\partial x^{\prime}}+v^{\prime} \frac{\partial u^{\prime}}{\partial y^{\prime}}=\mathrm{g} \beta\left(T^{\prime}-T_{\infty}^{\prime}\right)^{2}+\mathrm{g} \beta^{*}\left(C^{\prime}-C_{\infty}^{\prime}\right)-\frac{\sigma B_{0}{ }^{2}}{\rho} u^{\prime} \\
& u^{\prime} \frac{\partial T^{\prime}}{\partial x^{\prime}}+v^{\prime} \frac{\partial T^{\prime}}{\partial y^{\prime}}=\alpha \frac{\partial^{2} T^{\prime}}{\partial y^{\prime 2}} \\
& u^{\prime} \frac{\partial C^{\prime}}{\partial x^{\prime}}+v^{\prime} \frac{\partial C^{\prime}}{\partial y^{\prime}}=D \frac{\partial^{2} C^{\prime}}{\partial y^{\prime 2}}-k_{1}\left(C^{\prime}-C_{\infty}\right)
\end{aligned}
$$

The initial and boundary conditions are :

$$
\begin{aligned}
& \text { For } y^{\prime}=0: u^{\prime}=0, v^{\prime}=0, T^{\prime}=T_{w}{ }^{\prime}=A x^{n}, C^{\prime}=C_{w}{ }^{\prime} \\
& \text { For } y^{\prime} \rightarrow \infty,: u^{\prime}=u_{\infty}^{\prime}, T^{\prime} \rightarrow T_{\infty}{ }^{\prime}, C^{\prime} \rightarrow C_{\infty}{ }^{\prime}
\end{aligned}
$$

Here $v$ is the kinematic viscosity, $\mathrm{g}$ is the acceleration due to gravity, $\rho$ is the fluid density, $\beta$ is the volumetric coefficient of thermal expansion, $\beta^{*}$ is the volumetric coefficient of expansion with concentration, $\sigma$ is the electrical conductivity, $B_{0}$ is the magnetic field flux density, $D$ is mass diffusion coefficient, $\alpha$ is the thermal diffusivity and $k_{1}$ the chemical reaction coefficient. . Now we introduce the following dimensionless parameters:

$$
\begin{gathered}
\quad x=\frac{\chi^{\prime} u_{\infty}}{v}, y=\frac{y^{\prime} u_{\infty}}{v}, u=\frac{u^{\prime}}{u_{\infty}}, v=\frac{v^{\prime}}{u_{\infty}}, \theta=\frac{T^{\prime}-T_{\infty}^{\prime}}{T_{w}^{\prime}-T_{\infty}^{\prime}, \varphi=\frac{C^{\prime}-C_{\infty}^{\prime}}{C_{w}^{\prime}-C_{\infty}^{\prime}}} \\
G=\frac{v g \beta\left(T^{\prime}{ }_{w}^{\prime}-T_{\infty}^{\prime}\right)}{u_{\infty}{ }^{3}}, G^{*}=\frac{v g \beta^{*}\left(C_{w}^{\prime}-C_{\infty}^{\prime}\right)}{u_{\infty}{ }^{3}}, M=\frac{v \sigma B_{0}{ }^{2}}{\rho u_{\infty}{ }^{2}}, P r=\frac{v}{\alpha}, S C=\frac{v}{D}, \gamma=\frac{k_{1} v}{u_{\infty}{ }^{2}}
\end{gathered}
$$

With the help of these dimensionless parameters the governing equations (2)-(5) and the boundary conditions (6) take the form:

$$
\begin{aligned}
& \frac{\partial u}{\partial x}+\frac{\partial v}{\partial y}=0 \\
& u \frac{\partial u}{\partial x}+v \frac{\partial u}{\partial y}=\frac{\partial^{2} u}{\partial y^{2}}+G \theta^{2}+G^{*} \varphi-M u \\
& u \frac{\partial \theta}{\partial x}+v \frac{\partial \theta}{\partial y}=\frac{1}{P r} \frac{\partial^{2} \theta}{\partial y^{2}}
\end{aligned}
$$




$$
u \frac{\partial \varphi}{\partial x}+v \frac{\partial \varphi}{\partial y}=\frac{1}{S c} \frac{\partial^{2} \varphi}{\partial y^{2}}-\gamma \varphi
$$

The transformed boundary conditions are:

$$
\begin{aligned}
& u=0, v=0, \theta=1, \varphi=1 \text { at } y=0, t>0 \\
& u=0, v=0, \theta=0, \varphi=0 \text { as } y \rightarrow \infty, t>0
\end{aligned}
$$

Here $G$ is the thermal Grashof number, $G^{*}$ is the mass Grashof number, $M$ is the magnetic parameter,

$=11.4$ is the Prandtl number for water at $4{ }^{\circ} \mathrm{C}, \gamma$ is the chemical reaction parameter and $S c$ is the Schmidt number. Further we make use of the following similarity transformations in (7)-(11):

$$
\eta=y \sqrt{\frac{u_{\infty}}{v x}}, \psi(\eta)=\sqrt{u_{\infty} v x} f(\eta)
$$

The equations (7)-(11) are finally transformed to :

$$
\begin{aligned}
& f^{\prime \prime \prime}+\frac{1}{2} f f^{\prime}+\lambda_{1} \theta^{2}+\lambda_{2} \varphi-M f^{\prime}=0 \\
& \theta^{\prime \prime}+\frac{1}{2} P r . f \theta^{\prime}-n P r f^{\prime} \theta=0 \\
& \varphi^{\prime \prime}+\frac{1}{2} S c f \varphi^{\prime}-\gamma S c \cdot \operatorname{Re} . \varphi=0
\end{aligned}
$$

The boundary conditions are: $f=0, f^{\prime}=0, \theta=1, \varphi=1$ at $\eta=0$

$$
f^{\prime} \rightarrow 1, \theta \rightarrow 0, \varphi \rightarrow 0 \text { as } \eta \rightarrow \infty
$$

Here $R e=\frac{u_{00} x}{v}$ is the Reynolds number, $\lambda_{1}=\frac{G}{R e^{2}}$ and $\lambda_{2}=\frac{G^{*}}{R e^{2}}$ are respectively the thermal and mass buoyancy parameters of mixed convection. Dashes denote differentiation with respect to the similarity variable $\eta$. Knowing the velocity field, the physical quantities of interest to our study are the Skin friction $C_{f}=\frac{2 \tau_{w}}{\rho u^{2} \infty}$ which is given by the wall shear stress $\tau_{w}=\left[\mu\left(\frac{\partial u}{\partial y}\right)\right]_{y=0}$, the Local Nusselt number $N u=\frac{x q_{w}}{k\left(T_{w}-T_{\infty}\right)}$ given by the heat flux $q_{w}=-k\left(\frac{\partial T}{\partial y}\right)_{y=0}$ and the Local Sherwood number $S h=\frac{x M_{W}}{D\left(c_{w}-C_{\infty 0}\right)}$ given by the mass flux $M_{w}=-D\left[\frac{\partial C}{\partial y}\right]_{y=0}$.

In view of (12), the Skin Friction coefficient $C_{f}$ reduces to

$$
\frac{1}{2} C_{f}(R e)^{\frac{1}{2}}=f^{\prime \prime}(0)
$$

the Nusselt number $N u$ reduces to

$$
N u(R e)^{-\frac{1}{2}}=-\theta^{\prime}(0)
$$

the Sherwood number $S h$ reduces to

$$
\operatorname{Sh}(R e)^{-\frac{1}{2}}=-\varphi^{\prime}(0)
$$

\section{Solution Of The Problem:}

The non-linear coupled system of equations (13) - (15) along with the boundary conditions (16) are solved numerically using the continuation method with the help of the inbuilt Matlab routine which employs a fourth order Runge-Kutta algorithm for solving two point boundary value problems numerically. Computations are carried out for a fixed value of the buoyancy parameters $\lambda_{1}=\lambda_{2}=0.5$, different values of the exponent $\mathrm{n}$, the magnetic parameter $\mathrm{M}$ and the chemical reaction parameter $\gamma$. During computation, the Prandtl number $\operatorname{Pr}=11.4$, the Schimdt number $S c=500$ for an arbitrary species and Reynolds number $R e=10^{3}$ are chosen arbitrarily for water at $4^{\circ} \mathrm{C}$.We want to investigate the chemical reaction, plate temperature variation and magnetic effects on the flow field and as such keeping all other parameters fixed will highlight the effects clearly. Graphical results for $4^{0} \mathrm{C}$ are compared to the case of flow of water at $20^{\circ} \mathrm{C}$. The numerical values of $f^{\prime \prime}(0),\left\{-\theta^{\prime}(0)\right\}$ and $\left\{-\varphi^{\prime}(0)\right\}$ are evaluated and are listed in Table1. 
Chemical Reaction Effects On Mhd Mixed Convection Flow Of Water At Maximum Density Past A Vertical Plate Under Variable Temperature

\begin{tabular}{|c|c|c|c|c|c|c|c|c|c|c|}
\hline \multicolumn{11}{|c|}{ Table1 } \\
\hline \multicolumn{11}{|c|}{$\frac{G}{R e^{2}}=0.5=\frac{G^{*}}{R e^{2}}, S c=500, R e=10^{3}$} \\
\hline \multirow[b]{2}{*}{ M } & \multirow[b]{2}{*}{$\mathrm{n}$} & \multirow[b]{2}{*}{$\gamma$} & \multicolumn{3}{|c|}{ Water at $4^{0} \mathrm{C}$} & \multicolumn{3}{|c|}{ Water at $20^{\circ} \mathrm{C}$} & \multirow[b]{2}{*}{ Profile } & \multirow[b]{2}{*}{ Fig.no } \\
\hline & & & $f^{\prime \prime}(0)$ & $-\theta^{\prime}(0)$ & $-\varphi^{\prime}(0)$ & $f^{\prime \prime}(0)$ & $-\theta^{\prime}(0)$ & $-\varphi^{\prime}(0)$ & & \\
\hline 0 & 1 & 1 & 0.4754 & 1.3128 & \multirow{4}{*}{707.1068} & 0.5938 & 1.1946 & \multirow{4}{*}{707.1068} & \multirow{4}{*}{$\begin{array}{l}\text { Velocity } \\
\text { Temperature }\end{array}$} & \multirow{4}{*}{$\begin{array}{l}2 \\
3\end{array}$} \\
\hline 1 & 1 & 1 & 0.1908 & 0.7465 & & 0.2656 & 0.7640 & & & \\
\hline 2 & 1 & 1 & 0.1744 & 0.6681 & & 0.2287 & 0.6789 & & & \\
\hline 3 & 1 & 1 & 0.1633 & 0.6217 & & 0.2062 & 0.6251 & & & \\
\hline 1 & 0 & 1 & 0.2382 & 0.4697 & & 0.3143 & 0.4711 & \multirow{4}{*}{707.1068} & \multirow{4}{*}{$\begin{array}{l}\text { Velocity } \\
\text { Temperature }\end{array}$} & \multirow{4}{*}{$\begin{array}{l}4 \\
5\end{array}$} \\
\hline 1 & 1 & 1 & 0.1908 & 0.7465 & & 0.2656 & 0.7640 & & & \\
\hline 1 & 2 & 1 & 0.1715 & 0.8910 & 707.106 & 0.1987 & 0.8939 & & & \\
\hline 1 & 3 & 1 & 0.1598 & 0.9936 & & 0.1923 & 0.9799 & & & \\
\hline 1 & 1 & 0 & 0.2616 & 1.1383 & 2.0964 & 0.3590 & 0.8007 & 2.4923 & \multirow{4}{*}{$\begin{array}{l}\text { Velocity } \\
\text { Temperature } \\
\text { Concentration }\end{array}$} & \multirow{4}{*}{$\begin{array}{l}6 \\
7 \\
8\end{array}$} \\
\hline 1 & 3 & 1 & 0.1598 & 0.9936 & 707.106 & 0.2656 & 0.7640 & 707.1068 & & \\
\hline 1 & 1 & 2 & $0.190 d$ & 0.7465 & 1.0000 & 0.2654 & 0.7640 & 1.0000 & & \\
\hline 1 & 1 & 3 & 0.1908 & 0.7465 & 1.224 & 0.2653 & 0.7640 & 1.2247 & & \\
\hline
\end{tabular}

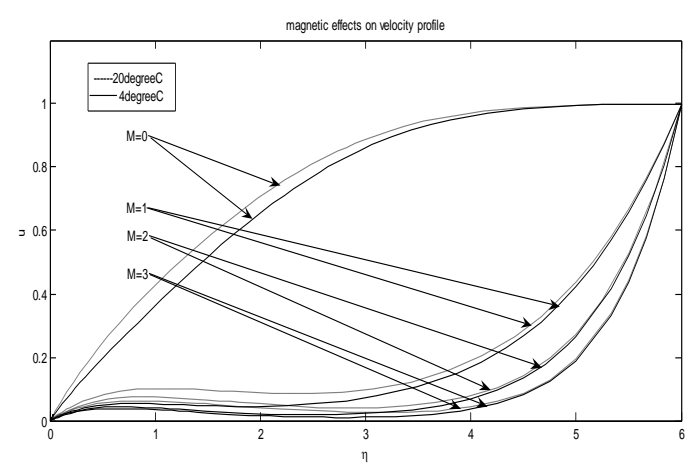

Figure2

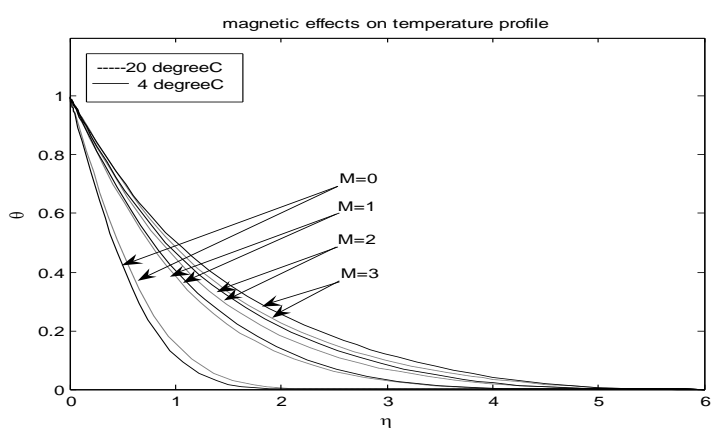

Figure3

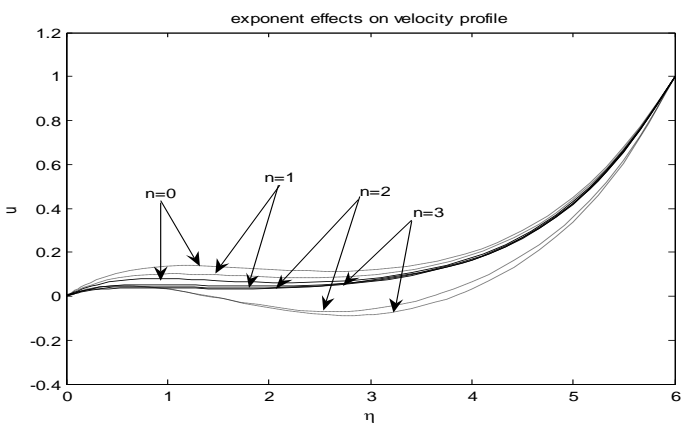

Figure4

www.iosrjournals.org 


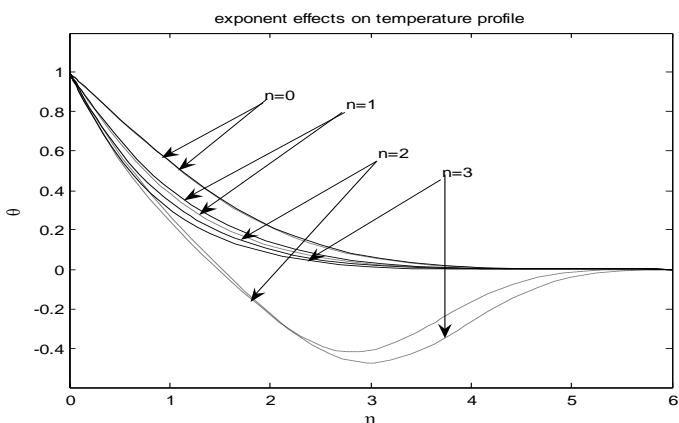

Figure5

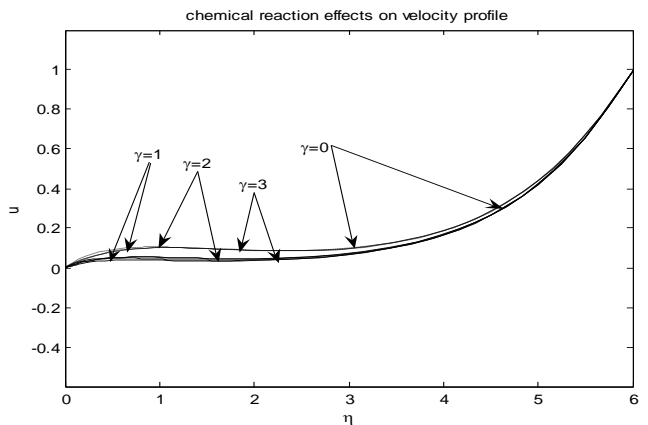

Figure6

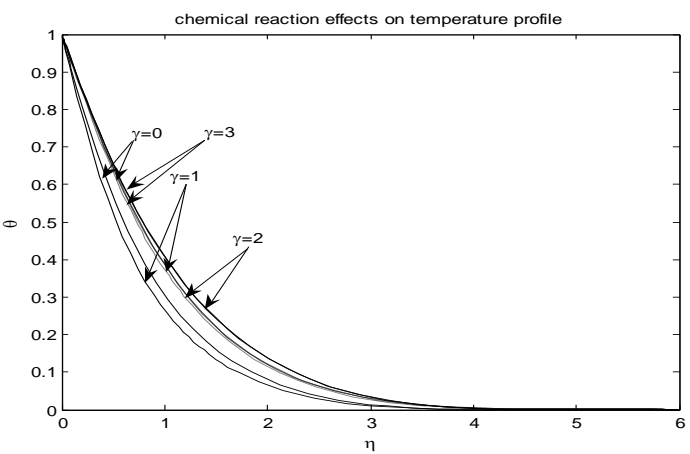

Figure7

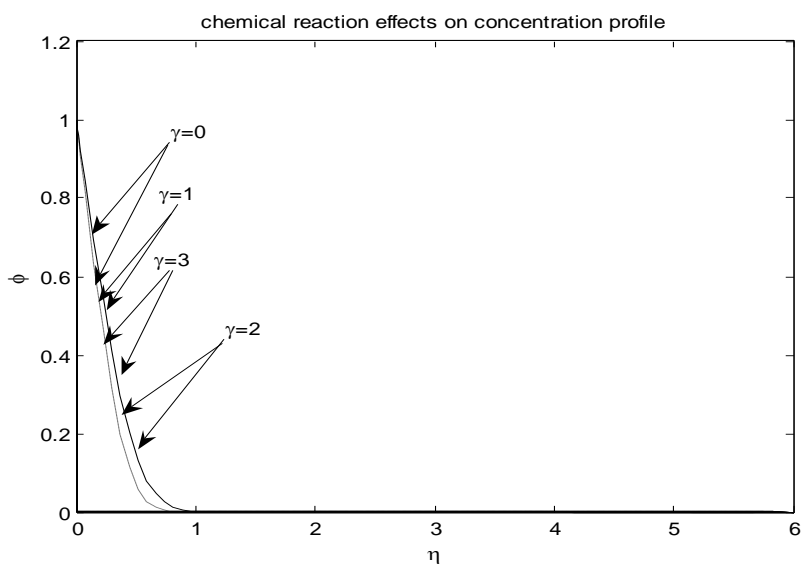

Figure8 


\section{CONCLUSIONS:}

(i) Magnetic effect on both the flow field is identical with a slight retarding effect on the velocity profile in the case of $4^{\circ} \mathrm{C}$.

(ii) In the absence of magnetic field the temperature profile for the flow of water at $4^{\circ} \mathrm{C}$ is low in comparison to the flow at $20^{\circ} \mathrm{C}$ but application of magnetic field has an enhancing effect on the flow for $4^{\circ} \mathrm{C}$ over $20^{\circ} \mathrm{C}$.

(iii) The effect of plate temperature variation has a significant effect on the velocity and temperature profiles. For constant temperature of the plate the temperature profiles are identical but as we increase the plate temperature, there is an enhancing effect on the temperature profile for the flow of water at $4^{\circ} \mathrm{C}$.

(iv) Temperature profile shows a pronounced variation for parabolic variation of the plate temperature in comparison to a linear variation in both the fields.

(iv) Presence of chemical reaction tend to reduce the velocity and temperature profile for $4^{\circ} \mathrm{C}$ in comparison to that of at $20^{\circ} \mathrm{C}$.

(v) There is no appreciable change in the concentration profile for both the flow fields.

\section{REFERENCES:}

[1] H. Schlichting and K. Gersten : Boundary Layer Theory ( $8^{\text {th }}$ ed.), Springer, 1999

[2] V.M. Soundalgekar, H.S.Takhar and N.V.Vighnesam (1988): Combined free and forced convection flow past a semi-infinite vertical plate with variable surface temperature: Nuclear Engineering anddesign:110, pp 95-98

[3] Graham Wilks (1973): Combined forced and free convection flow on vertical surfaces: Int. J. of Heat and Mass Transfer: 16, pp1958-1964

[4] M.S.Raju, X.Q.Liu and C.K. Law (1984): A formulation of combined forced and free convection past horizontal and vertical surfaces: Int. J. of Heat and Mass Transfer: 27(12), pp2215-2224

[5] U.N. Das, R.K. Deka and V.M. Soundalgekar (1998): Effect of mass transfer on flow past an impulsively started infinite vertical plate with chemical reaction: The Bulletin, GUMA,5, pp13-20

[6] A. Jyothi Bala and Vijaya Kumar Varma (2011): Unsteady MHD heat and mass transfer flow past asemi-infinite vertical porous moving plate with variable suction in the presence of heat generation and homogeneous chemical reaction: Int. J. of Appl. Math. And Mech., 7(7), pp20-24

[7] Mostafa AA Mahmoud (2007): A note on variable viscosity and chemical reaction effects on mixed convection heat and mass transfer along a semi-infinite vertical plate: Mathematical Problems in Engineering, 2007, pp1-7

[8] S. L. Goren (1966): On free convection in water at $4^{0} \mathrm{C}$ : Chemical Engineering Science, 21, pp515-518

[9] H. Herwig (1985): An asymptotic approach to free-convection flow at maximum density: Chemical Engineering Science, 40(9), pp1709-1715

[10] V.M. Soundalgekar, T.V. Ramana Murty and N.V. Vighnesam (1984): Combined forced and freeconvective flow of water at $4^{0} \mathrm{C}$ past a semi-infinite vertical plate: Int.J. of Heat \& Fluid Flow,5(1), pp54-56

[11] H.S.Takhar, P.C.Ram (1994): Magnetohydrodynamic free convection flow of water at $4^{0} \mathrm{C}$ through a porous medium: Int. Communications in Heat and Mass Transfer, 21(3), pp371-376

[12] N.V.Vighnesam \& V.M.Soundalgekar (1988): Combined free and forced convective flow of water at $4^{0} \mathrm{C}$ from a vertical plate with variable temperature, Indian J. of Engineering \& Materials Sciences, 5, pp124-126

[13] Md. Anwar Hossain, D.A.S. Rees (2005): Natural convection flow of water near its density maximum in a rectangular enclosure having isothermal walls with heat generation, Heat and Mass Transfer, 41,pp367-374

[14] M.F.Cawley, D.McGlynn, P.A.Mooney(2006): Measurement of the temperature of density maximum of water solutions using a convective flow technique, Int. J. of Heat and Mass Transfer, 49, pp1763-1772

[15] A. Pantokratoras (2004): Laminar natural convection in water near the density extremum along a vertical plate with sinusoidal surface temperature variation, Acta Mechanica, 172,pp211-218

[16] Waqar A. Khan, Rama Subba Reddy Gorla (2010): Mixed convection of water at $4^{0} \mathrm{C}$ along awedge with variable surface flux in a porous medium, Transport in Porous Media, 83, pp413-424

[17] S.C.Ling, R.Nazar, I.Pop, J.H.Merkin (2009): Mixed convection boundary layer flow in a porous medium filled with water close to its maximum density, Transport in Porous Media 76, pp139-151

[18] G.Palani (2008): Convection effects on flow past an inclined plate with variable surface temperatures in water at $4^{0} \mathrm{C}$, J. of Engineering-Annals of Faculty of Engineering Hunedoara, 6, pp75-82 